\title{
Linx
}

Revue des linguistes de l'université Paris X Nanterre

$54 \mid 2006$

La cause : approche pluridisciplinaire

\section{Ayres-Bennet, Wendy \& Carruthers, Janice, with Temple, Rosalind (2001) Problems and Perspectives. Studies in the modern french language}

Longman Linguistics Library, 406 pages

Danielle Leeman

\section{OpenEdition}

Journals

Édition électronique

URL : http://journals.openedition.org/linx/543

DOI : $10.4000 /$ linx.543

ISSN : 2118-9692

Éditeur

Presses universitaires de Paris Nanterre

Édition imprimée

Date de publication : 1 juin 2006

Pagination : 296-299

ISSN : 0246-8743

Référence électronique

Danielle Leeman, « Ayres-Bennet, Wendy \& Carruthers, Janice, with Temple, Rosalind (2001) Problems and Perspectives. Studies in the modern french language », Linx [En ligne], 54 | 2006, mis en ligne le 01 février 2007, consulté le 22 septembre 2020. URL : http://journals.openedition.org/linx/543 ; DOI : https://doi.org/10.4000/linx.543

Ce document a été généré automatiquement le 22 septembre 2020.

Département de Sciences du langage, Université Paris Ouest 


\title{
Ayres-Bennet, Wendy \& Carruthers, Janice, with Temple, Rosalind (2001) Problems and Perspectives. Studies in the modern french language
}

Longman Linguistics Library, 406 pages

\author{
Danielle Leeman
}

\section{RÉFÉRENCE}

Ayres-Bennet, Wendy \& Carruthers, Janice, with Temple, Rosalind (2001) Problems and Perspectives. Studies in the modern french language, Longman Linguistics Library, 406 pages.

L'ouvrage se présente comme un panorama de problèmes "intéressants " dans les domaines de la phonologie, de la morphologie, de la syntaxe et du lexique du français, avec chaque fois un intérêt particulier accordé à la variation et, lorsque c'est utile, un recours à la diachronie (par exemple pour vérifier l'affirmation selon laquelle la nonréalisation du ne négatif serait un fait récent). Il comporte deux parties d'inégale longueur: des Préliminaires (56 pages) et les questions traitées elles-mêmes. Les premiers définissent les notions et termes métalinguistiques (le français et les paramètres de la variation dans le premier cas, ce que l'on entend par "syllabe », " affixe ", " syntagme ", " clitique », " néologisme », etc. dans le second), présentent brièvement les différentes approches et écoles typiques de la linguistique française (structuralisme, grammaire générative, linguistique guillaumienne, "approche pronominale ») et justifient les choix opérés dans la deuxième partie à la fois pour les approches retenues et les problèmes abordés : l'objectif est de confronter le traitement de questions plutôt que de proposer une description complète du français; ainsi, le chapitre 6 n'est pas consacré aux temps verbaux en général mais se concentre sur la 
différenciation entre passé simple et passé composé; de même le chapitre 7 réduit le mode et la modalité à un examen approfondi du subjonctif.

2 Il est intéressant de se rendre compte, dans la section 3 de ces Préliminaires "Approaches and Schools", de ce qui est visible à l'international (en l'occurrence la Grande-Bretagne) de la linguistique française en langue française: d'une part des écoles «proprement françaises " (le modèle guillaumien, «l'approche pronominale ») et de l'autre l'inscription dans des courants internationaux (le structuralisme (Saussure, Bloomfield, Harris) et la grammaire générative). Pour ce qui concerne le structuralisme, sont à ce stade cités Martinet (phonologie et morphologie), Tesnière, Dubois et M. Gross (syntaxe) ; pour la grammaire générative dans les années soixantedix, Ruwet et Kayne (syntaxe), Guilbert (lexique); pour la linguistique énonciative Benveniste, Culioli, Ducrot, Anscombre et Ducrot, Kerbrat-Orecchioni, et pour la pragmatique du discours Roulet. Du côté des écoles purement françaises, Martin, Wilmet, Stéfanini sont cités comme les continuateurs de Guillaume, et deux pages sont consacrées à la présentation de l'approche pronominale (Blanche-Benveniste et autres).

Comme annoncé dans les Préliminaires, les chapitres de la deuxième partie sont chacun consacrés à un sujet sur lequel sont présentés différents traitements, par exemple la liaison (chapitre 1), les voyelles nasales (chapitre 2), le schwa ou « e muet » (chapitre 3) des points de vue du structuralisme, du générativisme, de la phonologie non linéaire, de la linguistique variationniste. La morphologie verbale (chapitre 4) montre de même les difficultés de segmentation et les désaccords auxquels elles donnent lieu (par exemple, Dubois considère trois formes vais, va/vas, vont tandis que M. Gross les analyse en $v$-ais/a/as/ont) et les divers classements de la conjugaison selon les critères: classification traditionnelle en trois groupes, sept conjugaisons chez Dubois, deux classes pour Van den Eynde \& Blanche-Benveniste, Plénat ou Gardes-Tamine. Le chapitre 5 , après avoir distingué entre temps et aspect, présente les différents aspects et les moyens de les distinguer puis les approches comparées de Brunot, Guillaume, Imbs, Reid, Martin, Wilmet - dont la classification est présentée comme la meilleure quoique complexe.

4 Le chapitre 6 , après un rappel de la manière dont les grammaires françaises présentent les temps du passé, se focalise sur un historique de leur formation puis sur les rapports entre passé simple et passé composé en français moderne, en particulier par le biais de Benveniste, Weinrich, Waugh et Monville-Burston, Vet. L'imparfait est présenté à travers les descriptions « reichenbachiennes» du type de celle de Molendijk, avec un paragraphe consacré à l' "imparfait narratif» et un autre aux temps composés et surcomposés. Le subjonctif (chapitre 7), de même, donne lieu à trois grandes questions : peut-on en rendre compte en termes sémantiques? Quelles sont les forces et les faiblesses des approches disponibles et quelles sont les explications les plus souvent retenues par les linguistes? Que dire de la vitalité du subjonctif compte tenu de son histoire (latin, ancien français, époque moderne), du point de vue de l'oral et de l'écrit et de ses variations sociolinguistiques et géographiques? Le chapitre 8 clôt le traitement du verbe avec un point sur la voix puis un développement sur les verbes pronominaux, toujours en faisant dialoguer différents points de vue, des grammaires traditionnelles aux approches transformationnelles en passant par les guillaumiens, avec une insistance particulière sur le passif pronominal et ses contraintes d'emploi.

5 Le chapitre 9 s'interroge sur la pertinence de considérer le français comme une langue «SVO ». Sont d'abord examinées les données historiques, puis celles du français 
moderne, où l'ordre en question est somme toute assez rare si l'on observe les grands corpus où fourmillent non seulement l'ordre SOV du fait de la complémentation clitique (Je le vois) mais aussi les détachements (Moi j'aime pas ça), les inversions (Sans doute allait-il mourir, Grande fut ma surprise), les constructions clivées (C'est le champagne que j'aime le plus), les différents types de topicalisation (Les poireaux je déteste). La conclusion est que le français est bien à ranger dans les langues SVO, les autres constructions correspondant à des choix marqués - issus de stratégies stylistiques ou pragmatiques. En 10 est abordée la question des relations entre phrases: subordination, coordination, parataxe; dans une première partie sont donnés des critères de définition permettant de différencier subordination et coordination, puis un sort particulier est réservé aux relatives « $\mathrm{du}$ français populaire »; la troisième section se pose la question d'une progression dans la « sophistication linguistique » allant de la parataxe à la subordination en passant par la coordination, à partir de ce qui ressort de l'observation de l'acquisition d'une part, de la variation sociolinguistique d'autre part.

6 Le chapitre 11 est consacré à la négation et récapitule les résultats des travaux les plus nombreux, qui ont été dévolus à la portée de la négation d'une part, à la restriction en ne...que d'autre part (où l'on peut regretter l'absence de l'apport d'Attal (1995) qui a remarqué que la construction n'est pas seulement restrictive, Il ne fait que dormir pouvant signifier «il passe son temps à dormir »), et l'absence de ne en français parlé enfin, avec un point historique et l'étude des différents facteurs susceptibles d'en être responsables (syntaxiques, phonétiques, sémantiques, stylistiques, sociolinguistiques). Les deux derniers chapitres sont consacrés respectivement aux néologismes considérés des points de vue interne et externe - les emprunts, leur intégration (phonologique, morphosyntaxique, sémantique), le statut des matériaux grecs et latins, l'attitude des Français face aux néologismes - et aux constructions de mot: les différents types de processus, la productivité selon les processus de formation, les travaux majeurs dans le domaine de la morphologie dérivationnelle (est présenté le modèle de Danielle Corbin).

7 Le livre se clôt par une grosse bibliographie de 26 pages, dont beaucoup de références en français et une bonne part sur l'analyse du français oral et la variation, un index des notions et un index des noms cités.

Il faut saluer le principe même de constitution de l'ouvrage, original relativement aux initiations existantes : ce n'est pas une grammaire en ceci que l'on ne cherche pas à présenter l'ensemble de la description syntaxique de la langue, et ce n'est pas un plaidoyer pro domo de tel cadre théorique dans le traitement de points particuliers du français. Un choix est fait dans les questions abordées, en tant qu'elles sont problématisées de différentes manières selon le paradigme de référence, ce qui permet au lecteur de saisir le lien entre cadre théorique, méthodologie, types d'observables, et de prendre conscience que tout point de vue rencontre des limites. La présentation est à la fois synthétique et claire, retenant les exemples les plus propres à faire saisir immédiatement les enjeux, et chaque chapitre se termine par une orientation du lecteur sur les travaux les plus propres à compléter la réflexion en même temps qu'ils le renseignent sur les sources de ce que l'on trouve exposé dans le chapitre. En bref, on ne saurait que recommander cet ouvrage, aux enseignants aussi bien qu'aux étudiants, quel que soit leur niveau (personnel ou académique). 


\section{BIBLIOGRAPHIE}

Attal, Pierre (1995) «Des mystères de ne que aux mystères de l'acceptabilité », in H. Bat-Zeev Shyldkrot \& L. Kupferman (éd.) Tendances récentes en linguistique française et générale. Volume dédié à David Gaatone, Amsterdam/Philadelphia, John Benjamins : 7-24. 